\title{
Antimicrobial Activity of a Series of 1-Alkyl-2-(4-Pyridyl) Pyridinium Bromides against Gram-Positive and Gram-Negative Bacteria
}

\author{
B.J. Denny ${ }^{a}$ L. Novotny ${ }^{a}$ P.W.J. West ${ }^{b} \quad$ M. Blesova ${ }^{c} \quad$ J. Zamocka ${ }^{d}$ \\ ${ }^{a}$ Department of Pharmaceutical Chemistry, Faculty of Pharmacy, ${ }^{b}$ Department of Medical Laboratory \\ Sciences, Faculty of Allied Health Sciences, Kuwait University, Kuwait; ${ }^{c}$ Faculty of Pharmacy, Veterinary and \\ Pharmaceutical University, Brno, Czech Republic, and ${ }^{\mathrm{d}}$ State Institute of Drug Control, Bratislava, Slovakia
}

\section{Key Words}

Bipyridyl · Alkyl chain - Methicillin-resistant

Staphylococcus aureus $\cdot$ Micelle $\cdot$ Efflux pumps

\begin{abstract}
Objective: To test a series of 1-alkyl-2-(4-pyridyl)pyridinium bromides with alkyl chains containing between 9 and 16 carbons against Gram-positive (Staphylococcus aureus) and Gram-negative (Escherichia coli, Stenotrophomonas maltophilia, Acinetobacter baumannii and Pseudomonas aeruginosa) bacteria. Materials and Methods: Chemical synthesis was based on the reaction of 2,4'-bipyridyl with alkyl bromide. Antimicrobial activity of the bipyridyls was measured by growing bacterial cultures on Mueller-Hinton agar in the presence and absence of inhibitors. Results: The compounds were most active against $S$. aureus. The most active compounds had alkyl chain lengths of between 11 and 16 carbons. Methicillin-sensitive $S$. aureus was more susceptible to the inhibitors than methicillin-resistant $S$. aureus

Preliminary results from this project were presented at the 13th ESCMID meeting in Glasgow, UK, May 2003 [West PWJ, Denny BJ, Novotny L, Blesova M, Zamocka J: Antimicrobial activity of 2,4'-bipyridyl ammonium salts. Clin Microbiol Infect 2003;9(suppl 1):810].
\end{abstract}

(C) $2005 \mathrm{~S}$. Karger $\mathrm{AG}$, Basel

$1011-7571 / 05 / 0146-0377 \$ 22.00 / 0$

Fax +4161306 1234 E-Mail karger@karger.ch www.karger.com
Accessible online at: www.karger.com/mpp
(MRSA). Two subclasses of MRSA existed which differed in their susceptibility to the inhibitors. The susceptibility of MRSA strains to the compounds was increased in the presence of the efflux pump inhibitor reserpine. The activity of the compounds against Gram-negative organisms was increased when the membrane-permeabilizing agent sodium citrate was introduced. Critical micelle concentrations of the compounds were much higher than minimum inhibitory concentrations of the inhibitors. Conclusion: The mechanism of action of the compounds may involve perturbing bacterial membranes. The resistance of some MRSA strains to the compounds may be related to efflux pumps.

Copyright (C) 2005 S. Karger AG, Basel

\section{Introduction}

The emergence of bacterial resistance to antibiotics and reduction in the activity of biocides is a major problem worldwide [1]. This limits the number of antimicrobial agents available to treat bacterial infections. As a result, new antibiotic agents are constantly being investigated to overcome this problem [2].

Quaternary ammonium salts with long alkyl chains have been shown to have a broad range of antimicrobial activity against Gram-negative and Gram-positive or- 
ganisms [3]. They are considered too toxic for systemic delivery in humans and animals but can be used topically. They have been used as biocides or disinfectants for many years $[4,5]$. The mechanism of action of these compounds is known to involve perturbing the bacterial cell membrane [6]. The antimicrobial effects are parabolically related to the length of the alkyl chains. Above and below certain chain lengths the agents are ineffective as antimicrobial agents [7].

1-alkyl-(pyridyl)pyridinium bromides (formerly known as bipyridyls) are a class of quaternary ammonium salts that have been shown to have herbicidal activity [8]. These compounds are toxic to humans. All of the bipyridyl compounds tested up to now are either 2,2'-bipyridyl derivatives (for example, diquat) or 4,4'-bipyridyl derivatives (for example, paraquat). Very little attention has been given to the biological and toxicological properties of 2,4'-bipyridyl compounds.

The aim of this study was to investigate the antimicrobial properties of a series of 1-alkyl-2-(4-pyridyl)pyridinium bromides (also known as 2,4'-bipyridyls) with varying lengths of alkyl chains (from C9 to C16). The structure of the compounds is shown in figure 1. The mechanism of the antimicrobial effect against Staphylococcus aureus was examined by adding the efflux pump inhibitor reserpine. The effect of a membrane permeabilizer on the activity of the compounds with Gram-negative organisms was also investigated. The correlation between length of the alkyl chains and micelle formation was examined.

\section{Materials and Methods}

\section{Synthesis of Compounds}

1-alkyl-2-(4-pyridyl)pyridinium bromides were prepared from 2,4'-pyridylpyridine and 1-bromoalkanes using the procedures previously described by Zamocka et al. [9]. 2,4'-pyridyl pyridine was reacted with 1-bromoalkane in molar ratio 0.02:0.05. The synthesis and purity of 1-alkyl-2-(4-pyridyl)pyridinium bromides was confirmed by elemental analysis, TLC, melting points and by UV and IR spectrophotometry. The substances were re-crystallized from dry acetone. TLC data were obtained with Merck Silica gel RP-8 F254S with fluorescent indicator. The solvent system used was pro-

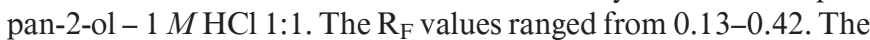
$\mathrm{R}_{\mathrm{F}}$ value for bipyridyl was 0.85 . The melting points of all the tested substances after re-crystallization were low and ranged between 36 and $38^{\circ} \mathrm{C}$ for the $\mathrm{C} 7$ compound to 68 and $70^{\circ} \mathrm{C}$ for the $\mathrm{C} 16 \mathrm{com}-$ pound. The parent unsubstituted bipyridyl had a melting point of $60-62{ }^{\circ} \mathrm{C}$. UV spectrophotometry yielded UV spectra with maximum absorbance at $294 \mathrm{~nm}$. The IR spectra contained a peak at or close to $1640 \mathrm{~cm}^{-1}\left(1635-1642 \mathrm{~cm}^{-1}\right)$ corresponding to the quaternary ammonium group.

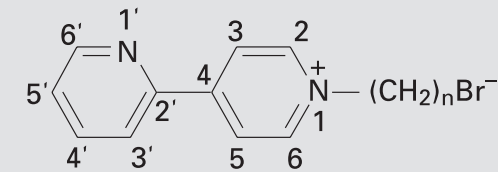

Fig. 1. Chemical structure of the 1-alkyl-2(4-pyridyl)pyridinium bromides tested $(\mathrm{n}=$ $7-14$ or 16$)$.

\section{Bacterial Isolates}

The strains of bacteria used were type strains from culture collections held in the Health Sciences Centre, Kuwait University, and clinical isolates from the Amiri Hospital, Kuwait. The cultures were stored in $10 \%$ skimmed milk (Oxoid, UK) at $-80^{\circ} \mathrm{C}$ and maintained on Mueller-Hinton agar (MH agar) (Acumedia, USA).

\section{Preparation of Media with Inhibitors}

Bipyridyl compounds were dissolved in dimethyl sulphoxide (Sigma, St. Louis, Mo., USA). Aliquots were added to MH agar (Acumedia), previously autoclaved and cooled to $50^{\circ} \mathrm{C}$. The media were dispensed in Petri dishes in 25-ml volumes. $\mathrm{MH}$ agars with dimethyl sulphoxide were used as growth controls.

\section{Determinations of Minimum Inhibitory Concentration}

Bacterial cultures grown overnight at $37^{\circ} \mathrm{C}$ on $\mathrm{MH}$ agar were suspended in peptone water to an optical density equivalent to a $0.5 \mathrm{McF}$ arland control (approx. $1.5 \times 10^{8}$ colony forming units $(\mathrm{CFU}) / \mathrm{ml}$ ). Drops of the suspensions (approx. $1 \mu \mathrm{l}$ ) were applied to the surface of the media containing appropriate concentrations of agents. The plates were incubated in air for $24 \mathrm{~h}$, at $37^{\circ} \mathrm{C}$ and examined for growth. The minimum inhibitory concentration (MIC) was taken as the lowest concentration of agent that inhibited visible growth.

\section{Determinations of MIC in the Presence of Membrane}

Permeabilizer or Reserpine

Bacterial suspensions were prepared as above and applied to media containing inhibitors as well as either $2.5 \%$ trisodium citrate or $20 \mathrm{mg} / \mathrm{l}$ reserpine (Sigma, in each case). These substances did not have antibacterial effects at these concentrations.

\section{Determinations of Critical Micelle Concentrations}

The critical micelle concentrations (CMCs) of the bipyridyl salts were determined using conductivity measurements as a function of concentration [10]. Conductivity measurements at $25^{\circ} \mathrm{C}$ were carried out using a Metrohm660 conductometer (Herisau, Switzerland) with two platinum electrodes (cell constant $\left.=0.79 \mathrm{~cm}^{-1}\right)$. The CMCs were determined by plotting the conductivity against the log of the concentration $(\mathrm{mol} / \mathrm{l})$. The $\mathrm{CMC}$ was noted as the sharp change in conductivity as the concentration of surface-active agent increased. 
Table 1. MICs (mg/l) of 1-alkyl-2-(4-pyridyl)pyridinium bromides (alkyl chain $=\mathrm{C} 12)$ against 13 strains of MSSA and 12 strains of MRSA, 9 strains of $S$. maltophilia, 10 strains of $A$. baumannii, 10 strains of $E$. coli and 10 strains of $P$. aeruginosa

\begin{tabular}{lrrrrrrrrr}
\hline & \multicolumn{1}{c}{ MIC number of strains } \\
\cline { 2 - 10 } & 2 & 4 & 8 & 16 & 32 & 64 & 128 & 256 & $>256$ \\
\hline MSSA & 13 & 0 & 0 & 0 & 0 & 0 & 0 & 0 & 0 \\
MRSA & 3 & 0 & 5 & 4 & 0 & 0 & 0 & 0 & 0 \\
S. maltophilia & 0 & 0 & 0 & 0 & 8 & 1 & 0 & 0 & 0 \\
A. baumannii & 0 & 0 & 0 & 0 & 7 & 3 & 0 & 0 & 0 \\
E. coli & 0 & 0 & 0 & 0 & 4 & 5 & 1 & 0 & 0 \\
P. aeruginosa & 0 & 0 & 0 & 0 & 0 & 0 & 0 & 2 & 8 \\
\hline
\end{tabular}

Table 2. MICs (mg/l) of 1-alkyl-2-(4-pyridyl)pyridinium bromides (alkyl chain $=$ C9-C16) against 13 strains of MSSA and 12 strains of MRSA

\begin{tabular}{lrrrrrrl}
\hline \multicolumn{7}{c}{ MIC number of strains } \\
\cline { 2 - 8 } & 2 & 4 & 8 & 16 & 32 & 64 & $>128$ \\
\hline MSSA & & & & & & & \\
C9 & 0 & 0 & 0 & 1 & 9 & 3 & 0 \\
C10 & 0 & 2 & 10 & 1 & 0 & 0 & 0 \\
C11 & 1 & 11 & 1 & 0 & 0 & 0 & 0 \\
C12 & 13 & 0 & 0 & 0 & 0 & 0 & 0 \\
C13 & 10 & 3 & 0 & 0 & 0 & 0 & 0 \\
C14 & 9 & 4 & 0 & 0 & 0 & 0 & 0 \\
C16 & 12 & 1 & 0 & 0 & 0 & 0 & 0 \\
\hline MRSA & & & & & & & \\
C9 & 0 & 0 & 0 & 1 & 2 & 1 & 8 \\
C10 & 0 & 0 & 3 & 0 & 1 & 5 & 3 \\
C11 & 0 & 3 & 0 & 0 & 2 & 7 & 0 \\
C12 & 3 & 0 & 5 & 4 & 0 & 0 & 0 \\
C13 & 3 & 0 & 2 & 4 & 3 & 0 & 0 \\
C14 & 3 & 0 & 2 & 7 & 0 & 0 & 0 \\
C16 & 1 & 2 & 1 & 0 & 8 & 0 & 0 \\
\hline
\end{tabular}

\section{Results}

\section{Antimicrobial Activity against Different Species}

The unsubstituted bipyridyl compound (no alkyl chain) had no appreciable antimicrobial activity against any of the organisms tested. The C7 and C8 alkyl derivatives had slight activity against $S$. aureus but no activity against Gram-negative bacteria.
Table 3. MICs (mg/l) of 1-alkyl-2-(4-pyridyl)pyridinium bromides (alkyl chain $=\mathrm{C} 10, \mathrm{C} 11, \mathrm{C} 12)$ against 12 strains of MRSA without reserpine and with $20 \mathrm{mg} / \mathrm{l}$ reserpine

\begin{tabular}{lcccccccc}
\hline \multicolumn{7}{c}{ MIC number of strains } \\
\cline { 2 - 9 } & 1 & 2 & 4 & 8 & 16 & 32 & 64 & $>128$ \\
\multicolumn{7}{l}{ MRSA } & without reserpine \\
C10 & 0 & 0 & 0 & 3 & 0 & 1 & 5 & 3 \\
C11 & 0 & 0 & 3 & 0 & 0 & 2 & 7 & 0 \\
C12 & 0 & 3 & 0 & 5 & 4 & 0 & 0 & 0 \\
C13 & 0 & 3 & 0 & 4 & 3 & 0 & 0 & 0 \\
C14 & 0 & 3 & 0 & 2 & 7 & 0 & 0 & 0 \\
\hline MRSA & with & reserpine & & & & & & \\
C10 & 0 & 3 & 8 & 1 & 0 & 0 & 0 & 0 \\
C11 & 0 & 3 & 2 & 1 & 5 & 0 & 1 & 0 \\
C12 & 3 & 0 & 1 & 8 & 0 & 0 & 0 & 0 \\
C13 & 0 & 3 & 1 & 8 & 0 & 0 & 0 & 0 \\
C14 & 0 & 0 & 4 & 8 & 0 & 0 & 0 & 0 \\
\hline
\end{tabular}

The sensitivity of different species of bacteria to the C12 bipyridyl compound is shown in table 1. The inhibitor is more active against $S$. aureus than Gram-negative bacilli. Methicillin-sensitive strains of $S$. aureus (MSSA) were more susceptible to the bipyridyl compounds than most methicillin-resistant strains (MRSA).

\section{Antimicrobial Activity as a Function of Alkyl Chain Length versus MSSA and MRSA}

The variation of MIC versus alkyl chain length (C9C16) against 12 strains of MSSA plus the ATCC25923 control strain of $S$. aureus and 12 strains of MRSA is shown in table 2 .

The MICs for MSSA exhibited a plateauing effect with alkyl chain length with the maximum activity being for C12-C16 alkyl chains.

Two subpopulations of MRSA were observed. Three of the 12 strains tested had the same susceptibility to the bipyridyls as MSSA. These strains also exhibited a plateauing effect of activity with increasing alkyl chain length. The other nine MRSA strains tested exhibited a parabolic relationship between MIC and alkyl chain length. Maximum activity was observed with the $\mathrm{C} 12$ compound.

\section{Inhibition of Efflux Pumps}

The effect on the MIC of the alkaloid reserpine is shown in table 3. The greatest reduction in MIC was observed for the $\mathrm{C} 10$ inhibitor. Reserpine has a smaller effect on MIC as the chain length of the bipyridyl increases. 
Table 4. MICs (mg/l) of 1-alkyl-2-(4-pyridyl)pyridinium bromides $($ alkyl chain $=\mathrm{C} 12)$ against $S$. maltophilia, A. baumannii, E. coli, $P$. aeruginosa without sodium citrate and with $2.5 \%$ sodium citrate

MIC number of strains

$\begin{array}{lllllllll}1 & 2 & 4 & 8 & 16 & 32 & 64 & 128 & \geq 256\end{array}$

Without permeabilizer

$\begin{array}{lllllllllr}\text { S. maltophilia } & 0 & 0 & 0 & 0 & 0 & 8 & 1 & 0 & 0 \\ \text { A. baumannii } & 0 & 0 & 0 & 0 & 0 & 7 & 3 & 0 & 0 \\ \text { E. coli } & 0 & 0 & 0 & 0 & 0 & 4 & 5 & 1 & 0 \\ P . \text { aeruginosa } & 0 & 0 & 0 & 0 & 0 & 0 & 0 & 0 & 10\end{array}$

With permeabilizer

$\begin{array}{llllllllll}\text { S. maltophilia } & 0 & 0 & 8 & 1 & 0 & 0 & 0 & 0 & 0\end{array}$

$\begin{array}{llllllllll}\text { A. } \text { baumannii }^{\text {a }} & 1 & 3 & 5 & 0 & 0 & 0 & 0 & 0 & 0\end{array}$

$\begin{array}{llllllllll}\text { E. coli } & 0 & 0 & 10 & 0 & 0 & 0 & 0 & 0 & 0\end{array}$

$\begin{array}{lllllllllll}P . \text { aeruginosa }^{\mathrm{b}} & 0 & 0 & 1 & 0 & 0 & 0 & - & - & -\end{array}$
lizer.

a One strain of $A$. baumannii was inhibited by the permeabi-

${ }^{\mathrm{b}}$ For $P$. aeruginosa the highest inhibitor concentration tested was $32 \mathrm{mg} / \mathrm{l}$. Nine strains had MIC > $32 \mathrm{mg} / \mathrm{l}$.

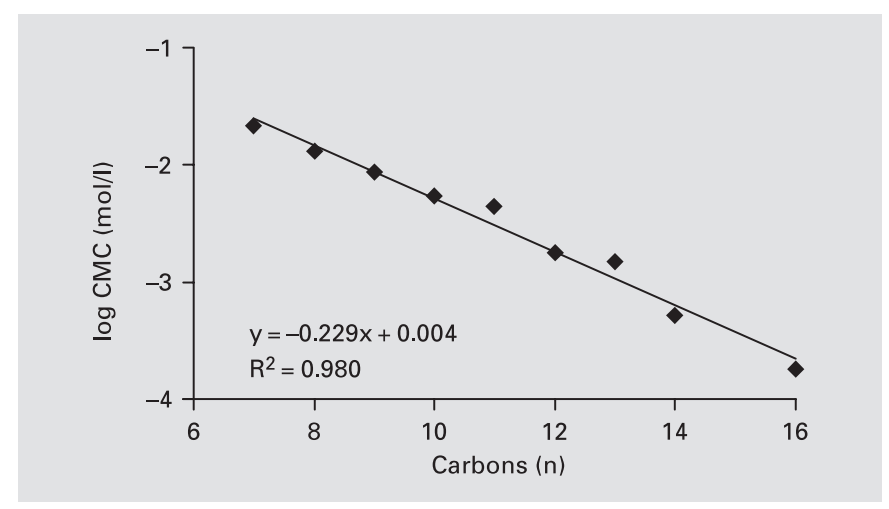

Fig. 2. Relationship between $\log \mathrm{CMC}$ and the number of carbons in the alkyl chain of 1-alkyl-2-(4-pyridyl)pyridinium bromides.

\section{Effect of the Membrane Permeabilizer Sodium}

\section{Citrate on the MIC Values for Selected Organisms}

The Gram-negative organisms (Escherichia coli, Pseudomonas aeruginosa, Stenotrophomonas maltophilia) have low susceptibility to the bipyridyls but become more susceptible when the membrane permeabilizer sodium citrate is introduced (table 4). In the absence of a membrane permeabilizer $P$. aeruginosa was completely resistant to the bipyridyls. In the presence of the permeabilizer, the organism showed some sensitivity.

\section{Micelle Formation}

The linear relationship between $\log \mathrm{CMC}$ and $\mathrm{N}$, the number of carbons in the alkyl chain is shown in figure 2 . $\log C M C=-0.229 \mathrm{~N}+0.004\left(\mathrm{R}^{2}=0.980\right) . \log C M C$ is usually considered a relative measure of the hydrophobicity of a surfactant.

\section{Discussion}

The compounds tested behaved similarly to quaternary ammonium compounds. Gram-positive organisms, such as $S$. aureus, are known to be more susceptible than Gram-negative organisms [11]. P. aeruginosa is the most resistant species. This has been shown previously [12].

The MICs for the MSSA strains were very similar to those previously reported for the 2,2'-bipyridyl series [13]. That group also observed the plateauing effect with maximal activity for the $\mathrm{C} 12$ to $\mathrm{C} 16$ alkyl chains. A parabolic variation of MIC versus alkyl chain length was observed for nine of the 12 MRSA strains (table 2). Other groups have also observed a parabolic relationship between length of alkyl chain and biological activity for a series of surfactants and suggested that it is related to micelle formation $[14,15]$. As the length of an alkyl chain increases, the tendency to form micelles also increases.

For both MSSA and MRSA, the MICs were much lower than the CMCs. Alternative CMC determinations using a glass stalagmometer to measure surface tension as a function of surfactant concentration gave very similar results. Thus, the increase in MICs for longer chain compounds for the MRSA strains is probably not related to micelle formation.

Apart from micelle formation, other theories have been proposed to explain the parabolic relationship between length of alkyl chain of a surfactant and its biological activity. These include limited volume at the site of action in the membrane, phase transitions at the lipid bilayers, or changes in stereochemistry or membrane solubility of the surfactant. Balgavy and Devinski have reviewed these theories [16].

The reduced susceptibility of $S$. aureus to quaternary ammonium compounds, such as the disinfectants benzalkonium chloride or cetylpyridinium chloride, is thought to involve multidrug efflux pumps encoded by plasmidmediated $q a c A$ and $q a c B$ genes $[17,18]$. These genes are more commonly found in MRSA than in MSSA [19]. We observed two populations of MRSA in terms of susceptibility to the inhibitors. This may be related to the presence of the above genes. Reserpine has been shown to 
inhibit efflux pumps in $S$. aureus [20,21]. Our data show that the MICs of shorter chain inhibitors $(\mathrm{C} 10, \mathrm{C} 11)$ were significantly reduced in the presence of reserpine. For the longer chain inhibitors $(\mathrm{C} 13, \mathrm{C} 14)$ the effect was less pronounced, suggesting that the efficiency of the efflux pump may decrease as alkyl chain length increases.

The outer membrane of Gram-negative bacteria is surrounded by a lipopolysaccharide surface. This outer membrane barrier is one of the factors that explain the greater resistance of Gram-negative organisms to antibiotics compared with Gram-positive organisms. Grampositive organisms have a much simpler cell wall composed primarily of peptidoglycan (a polysaccharide) and teichoic acid. A variety of agents can permeabilize Gramnegative membranes and make them more susceptible to antibiotics or other agents [22, 23]. Generally, these are anionic substances that chelate metal ions such as $\mathrm{Ca}^{2+}$ or $\mathrm{Mg}^{2+}$. These ions are necessary to stabilize the lipopolysaccharide surface.

\section{Conclusion}

Our results show that 2,4'-bipyridyl ammonium salts are effective agents against most $S$. aureus strains. Reduced susceptibility in some MRSA strains may be related to an efflux mechanism. The addition of a membrane permeabilizer increases the susceptibility of Gramnegative bacteria showing the outer layer of these organisms is a barrier to these compounds. This supports the hypothesis that they inhibit bacteria by causing perturbations of the cell membrane.

\section{Acknowledgments}

M.B. received support from The Ministry of Education, Youths and Sport of the Czech Republic No. 163700003.

We thank Dr. Edet Udo, Department of Microbiology, Faculty of Medicine, Kuwait University for supplying strains of methicillin-resistant $S$. aureus and for useful discussions.

\section{References}

$\checkmark 1$ Normark BH, Normark S: Evolution and spread of antibiotic resistance. J Intern Med 2002;252:91-106.

2 Chopra I, Hesse L, O’Neill AJ: Exploiting current understanding of antibiotic action for discovery of new drugs. J Appl Microbiol 2002; 92(suppl):4S-15S

3 Birnie CR, Malamud D, Schnaare RL: Antimicrobial evaluation of $\mathrm{N}$-alkyl betaines and $\mathrm{N}$ alkyl-N,N-dimethylamine oxides with variations in chain length. Antimicrob Agents Chemother 2000;44:2514-2517.

$\checkmark 4$ Jacobs WA: The bactericidal properties of the quaternary salts of hexamethylenetetramine I. J Exp Med 1916;23:563-568.

$\checkmark 5$ Dixon RE, Kaslow RA, Mackel DC, Fulkerson CC, Mallison GF: Aqueous quaternary ammonium antiseptics and disinfectants. JAMA 1976;236:2415-2417.

6 McDonnell G, Russell AD: Antiseptics and disinfectants: activity, action, and resistance. Clin Microbiol Rev 1999;12:147-179.

$>7$ Devinsky F, Kopecka-Leitmanova A, Sersen F, Balgavy P: Cut-off effect in antimicrobial activity and in membrane perturbation efficiency of the homologous series of N, N-dimethylamine oxides. J Pharm Pharmacol 1990;42:790-794.

8 Akhavein AA, Linscott DL: The dipyridylium herbicides, paraquat and diquat. Residue Rev 1968;23:97-145.

9 Zamocka J, Lacko I, Devinsky F: Synthesis, physico-chemical and antimicrobial activities of 1-alkyl-2-(pyridyl)pyridinium bromides. Pharmazie 1994;49:66-67.
0 Nakamura H, Sano A, Matsuura K: Determination of critical micelle concentration of anionic surfactants by capillary electrophoresis using 2-naphthalenemethanol as a marker for micelle formation. Anal Sci 1998;14:379382.

11 Russell AD: Bacterial adaptation and resistance to antiseptics, disinfectants and preservatives is not a new phenomenon. J Hosp Infect 2004;57:97-104.

12 Lambert PA: Mechanisms of antibiotic resistance in Pseudomonas aeruginosa. J R Soc Med 2002;95(suppl 41):22-26.

13 Devinsky F, Zamocka J, Lacko I, Polakovicova M: QSAR and CAMM study of amphiphilic antimicrobially active $2,2^{\prime}$-bipyridyl monoammonium salts. Pharmazie 1996;51:727-731.

14 Kopecky F: Micellization and other associations of amphiphilic antimicrobial quaternary ammonium salts in aqueous solutions. Pharmazie 1996;51:135-144.

15 Tomlinson E, Brown MR, Davis SS: Effect of colloidal association on the measured activity of alkylbenzyldimethyl-ammonium chlorides against Pseudomonas aeruginosa. J Med Chem 1977;20:1277-1282.

16 Balgavy P, Devinsky F: Cut-off effects in biological activities of surfactants. Adv Colloid Interface Sci 1996;66:23-63.

17 Suller MTE, Russell AD: Antibiotic and biocide resistance in methicillin-resistant Staphylococcus aureus and vancomycin-resistant Enterococcus. J Hosp Infect 1999;43:281-291.
18 Townsend DE, Greed L, Ashdown N, Grubb WB: Plasmid-mediated resistance to quaternary ammonium compounds in methicillinresistant Staphylococcus aureus. Med J Aust 1983;2:310.

19 Alam MM, Kobayashi N, Uehara N, Watanabe N: Analysis on distribution and genomic diversity of high-level antiseptic resistance genes qacA and qacB in human clinical isolates of Staphylococcus aureus. Microb Drug Resist 2003;9:109-121.

20 Gibbons S, Udo EE: The effect of reserpine, a modulator of multidrug efflux pumps, on the in vitro activity of tetracycline against clinical isolates of methicillin resistant Staphylococcus aureus (MRSA) possessing the tet(K) determinant. Phytother Res 2000;14:139-140.

-21 Schmitz FJ, Fluit AC, Luckefahr M, Engler B, Hofmann B, Verhoef J, Heinz HP, Haddig U: The effect of reserpine, an inhibitor of multidrug efflux pumps, on the in vitro activities of ciprofloxacin, sparfloxacin and moxifloxacin against clinical isolates of Staphylococcus aureus. J Antimicrob Chemother 1998;42:807810

22 Denny B, West P, Panigrahi D: Effects of permeabilizers on antimicrobial susceptibility of Stenotrophomonas maltophilia and Acinetobacter spp. J Microbiol Immunol Infect 2003; 36:72-76.

23 Vaara M: Agents that increase the permeability of the outer membrane. Microbiol Rev 1992;56:395-411. 